\title{
Utilizing NMR Mud Logging Technology To Measure Reservoir Fundamental Parameters in Well Site
}

\author{
Yingzhao Zheng ${ }^{1,2}$, Dehui Wan', Muhammad $\mathrm{Ayaz}^{2}$, Caiqin $\mathrm{Ma}^{3}$ \\ ${ }^{1}$ School of Energy Resources, China University of Geosciences, Beijing, 100083, China \\ ${ }^{2}$ Mud Logging International Department, Great wall drilling company, Beijing, 100101, China \\ ${ }^{3}$ Beijing Winfield Petroleum Technology Company, Beijing, 100083, Chin \\ Email: zhengyingzhao@cnlc.cn
}

Received 2013

\begin{abstract}
Nuclear Magnetic Resonance mud logging technology (NMR mud logging) is a new mud logging technology. Mainly applies the CPMG (Carr-Purcell-Meiboom-Gill) pulse sequence to measure transverse relaxation time $\left(\mathrm{T}_{2}\right)$ of the fluid. NMR mud logging can measure drill cutting, core and sidewall core in the well site, also according to the experiment results, the sample type and size has little effect to analysis result. Through NMR logging, we can obtain several petrophysical parameters such as total porosity, effective porosity, permeability, oil saturation, water saturation, movable fluid saturation, movable oil saturation, movable water saturation, irreducible fluid saturation, irreducible oil saturation, irreducible water saturation, pore size and distribution in rock samples, etc. NMR mud logging has been used nearly 10 years in China, Sudan, Kazakhstan, etc. it plays an important role in the interpretation and evaluation of reservoir and its fluids.
\end{abstract}

Keywords: NMR Mud Logging; Porosity; Oil Saturation; Reservoir Fluids; T2 Cutoff; Spectrum

\section{Introduction}

Faster drilling speed and higher drilling cost (especially offshore) dictate reducing cost and decreasing the time for reservoir evaluation. Simultaneously, it is necessary to ensure the reliability of the results of such evaluation. A number of techniques which analyze drill cuttings at the well site for quantitative formation evaluation have been developed, among them, NMR mud logging is the best to evaluate the reservoir.

Porosity, permeability and oil saturation are fundamental parameters for reservoir evaluation. In order to get these petrophysical parameters of the reservoir, we usually analyze the core samples in the laboratory, but that need a long period and cost a lot. As the drilling cost increase, there is an urgent requirement to measure fundamental parameters of the reservoir in the exploration and production of oil and gas field, especially that of low porosity and low permeability reservoir and hiding reservoir.

NMR mud logging technology has implemented the transfer from the lab to the well drilling field for the petrophysical analysis of the conventional core, and expanded the analysis object from conventional core to the cutting and sidewall core.
NMR mud logging technology utilizes hydrogen atomic nucleus $\left({ }^{1} \mathrm{H}\right)$ rich in the formation fluid (oil, gas and water) to obtain the petrophysical parameter such as the porosity, permeability, fluid saturation, fluid property, movable fluid and irreducible fluid [1-4]. This technology has been widely applied to the evaluation of reservoir and its fluids.

It has many merits, such as little amount of sample used, fast analysis speed, several parameters from one sample, high accuracy, high continuity and analysis while drilling. It has significance in classifying and evaluating effective reservoir, instructing drilling on site and providing data for the well completion discussion and drilling completion test, so it has great role in the exploration and the production of oil and natural gas.

In 2001, A portable NMR rock sample analyzer was developed by NMR Laboratory of RIPED (Research Institute of Petroleum Exploration and Development, Petrochina), China. The researchers performed much basic work on the analysis of core and cuttings, confirmed the feasibility of NMR analysis of cuttings, conducted the field test of the new NMR logging technology in several Chinese oilfields, and successfully solved many critical problems. NMR mud logging has exerted important role in the exploration and production of oil and gas field. 


\section{NMR Mud Logging Methods and Parameters}

NMR mud logging unit (use RecCore 2500 unit as an example, the picture is show in Figure 1) use one big probe $(25 \mathrm{~mm}$ in diameter) and one small $(10 \mathrm{~mm}$ in diameter) probe. The big probe can used to analyze the samples of side wall core and conventional core; the small probe can be used to analyze cutting samples.

\subsection{NMR $T_{2}$ Spectrum and $T_{2}$ Cutoff Value}

After the samples are well treated, they can be analyzed. NMR mud logging first obtains the echo string, which can be mathematically inversed to get $T_{2}$ spectrum. Figure 2 shows the NMR $T_{2}$ spectrum of a sample. The $\mathrm{x}$-coordinate represent the $\mathrm{T}_{2}$ relaxation time, the ordinate represent amplitude, which is in direct proportion to the fluid amount with certain $\mathrm{T}_{2}$ relaxation time [5].

As NMR $T_{2}$ relaxation time reflects the interface strength of the pore solid surface to the fluids it contained. The fluid with more $T_{2}$ relaxation time (fluids in big pore does not contact closely with solid surface) is the movable fluid or free fluid. The fluid with less $T_{2}$ relaxation time (fluids in small pore contact closely with solid surface) is the irreducible fluid. The boundary between the movable fluid and the irreducible fluid in $T_{2}$ spectrum is called as movable fluid $\mathrm{T}_{2}$ cutoff value (shown as Figure 3 ). The $\mathrm{T}_{2}$ cutoff value vary from fields to fields, but mainly distributes between $8 \sim 30 \mathrm{~ms}$. It can be accurately measured by the indoor centrifugal method [6].

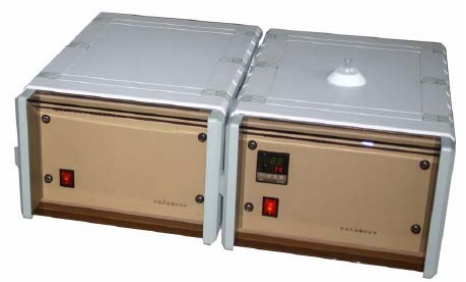

Figure 1. NMR mud logging unit.

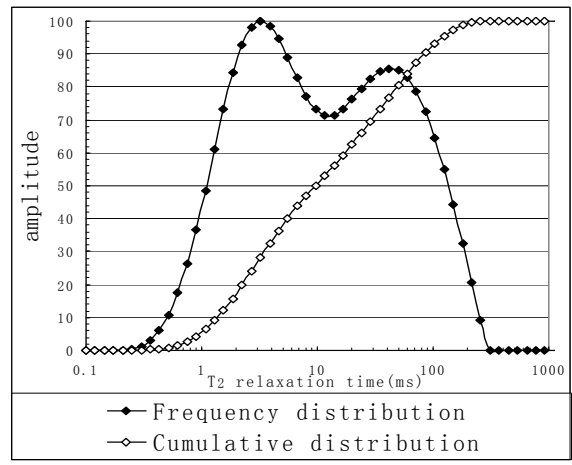

Figure 2: NMR mud logging $T_{2}$ spectrum.
The main NMR measurement parameters:

Porosity $(\Phi)$

Permeability (K)

Oil Saturation (So)

Free Fluid Saturation (Sm)

Free Water Saturation (Swm)

Irreducible Water Saturation (Swi)

\subsection{NMR Porosity Measuring Principle and Method}

The NMR signal amplitude is proportional to the fluid amount contained in rock sample, when the samples are fully saturated with fluids (water or oil), we can measure the total porosity. When the samples are centrifuged, all the movable fluid will be removed, only the irreducible fluid (clay bound fluid) are remained in the sample, so the effective porosity can be measured.

NMR porosity measuring steps are as follows: Firstly, calibrate the NMR mud logging unit, establish the linear relationship between the NMR signal amplitude and the porosity (shown as Figure 4); Then measure NMR sample, get sample NMR signal amplitude; At last, substitute the signal amplitude into sample porosity.

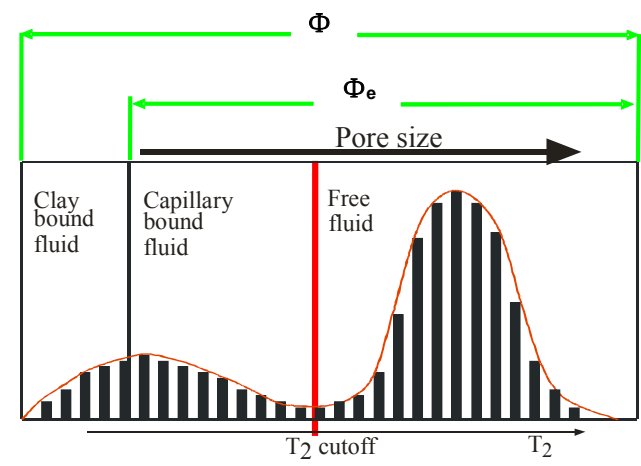

Figure 3. Relationship between porosity and effective porosity.

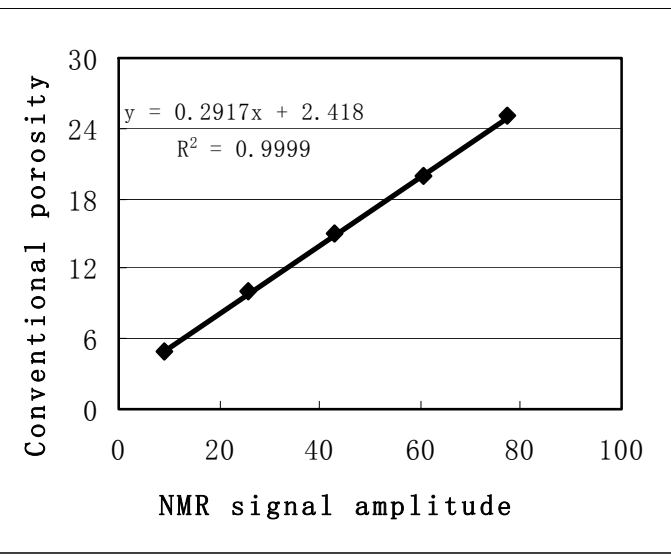

Figure 4. The linear relationship between NMR signal amplitude and the porosity. 


\subsection{NMR Saturation Measuring Principle and Method}

In order to measure oil saturation, the sample needs to be analyzed twice. Firstly, analyze the sample under the condition that the pore space is fully saturated by water, and we measure the total signal of oil and water; secondly, we dip that sample in manganese $\mathrm{Mncl}_{2}$ (concentration $10000 \mathrm{mg} / \mathrm{l}$ ), which is a paramagnetic substance to eliminate water signal. Then, we analyze that $\mathrm{Mncl}_{2}$ saturated sample to obtain oil signal (Figure 5).

When we get both of the $T_{2}$ spectrums like Figure 5, we can derive the oil saturation by following calculation.

$$
\mathrm{S}_{\mathrm{o}}=\frac{\int_{0}^{\mathrm{T}_{2 \max }} \mathrm{A}_{\mathrm{o}}(\mathrm{t}) \mathrm{dt}}{\int_{0}^{\mathrm{T}_{2 \max }} \mathrm{A}_{\mathrm{ow}}(\mathrm{t}) \mathrm{dt}} \times 100 \%
$$

where, $S_{o}$ is oil saturation, $A_{o w}$ represents oil and water signal amplitude, $A_{0}$ represents oil signal amplitude.

Meanwhile, other parameters, such as movable oil saturation, irreducible oil saturation, movable water saturation and irreducible water saturation (Xiao, et al., 2000) can be got.

\subsection{NMR Permeability Calculating Principle and Method}

NMR permeability can be calculated by an empirical model--Timmur-Coates model.

$$
\mathrm{K}_{\mathrm{nmr}}=\left(\frac{\phi_{\mathrm{nmr}}}{\mathrm{C}}\right)^{4} \cdot\left(\frac{\mathrm{Sm}}{1-\mathrm{Sm}}\right)^{2}
$$

where, $\mathrm{K}_{\mathrm{nmr}}-\mathrm{NMR}$ permeability, $10^{-3} \mu \mathrm{m}^{2}$;

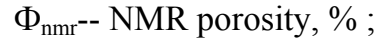

$\mathrm{S}_{\mathrm{m}}$--Free Fluid Saturation, \%;

$\mathrm{C}$-regional coefficient, Changes with strata, determined by core analysis.

\subsection{Pore Structure Evaluation}

NMR $T_{2}$ spectrum depend on topology of pores (shape and size); In large pores, $\mathrm{T}_{2}$ value are longer, as more nuclei are available in the pores; In small pores, $T_{2}$ value are short. The distribution of $T_{2}$ spectrum can range from

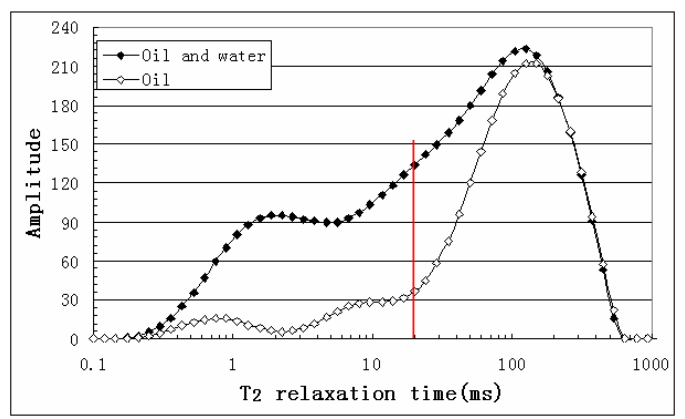

Figure 5. $T_{2}$ spectrum separation of oil and water.
1 to $10,000 \mathrm{~ms}$. Therefore, $\mathrm{T}_{2}$ is in direct proportion to pore throat radius. Generally, wider distribution range of $\mathrm{T}_{2}$ spectrum and lower amplitudes represent poor sorting of pores, and smaller pore throat (He et al., 2005). In addition, higher amplitudes for higher $T_{2}$ cutoff values represent larger pores, and thus the reservoir formation is potentially better.

Different samples have different pore structure, and the same for the lithology. These differences lead to the difference of $T_{2}$ differential curves and cumulative curves. Therefore, the size, sort and distribution of pore throats can be evaluated according to $T_{2}$ curves of saturated samples.

\section{NMR Mud Logging Application}

By comprehensive use of NMR mud logging data, the geologists can quickly evaluate the reservoir and its fluids in well site, which will be very useful for the discussion of well completion, perforation and well test $[7,8]$.

NMR mud logging technology has been widely used more than 10 years around the world by Chinese mud logging companies. A few thousands of cuttings and core samples have been measured by the NMR mud logging system and excellent application results of this technology have been achieved. Now, it plays an important role in the interpretation and evaluation of reservoir and its fluids.

\subsection{The Application of NMR mud Logging in BA-1, Nanyang oil Field, China}

Ba-1 is a wildcat well, located in Henan province, China. NMR mud logging analyzed 13 samples in the interval $4044-4047 \mathrm{~m}$, which is best fluorescence show interval of the well.

In that interval, the lothology is fine sandstone with good oil shows. For chromatograph logging, the maxim total gas is $6.9 \%$, also the trip gas are very strong, even lasts $25 \mathrm{~min}$.Wireline logging interpreted this interval as pay zone.

NMR logging result are as follows: Porosity: 7.05\%-8. 20\%; Permeability:0. 01-0.18 $\times 10^{-3} \mu \mathrm{m}^{2}$; Oil Saturation: 5.47\%-8.98\%; Free Fluid Saturation: 26.01\%-33. 84\%; Free Water Saturation: 24.06\%-30.21\%; Irreducible Water Saturation: 61.25-70. 47\%; NMR interpreted this interval as dry zone.

This interval was tested, but the maxim output is only $0.51 \mathrm{t} / \mathrm{d}$. NMR mud logging interpretation coincides with test result.

\subsection{The Application of NMR Logging Technology in a Well of Jilin oil Field, China}

In a well of Jilin oil field, NMR mud logging analyzed 47 cuttings samples. Each cuttings sample obtained the 
Table 1. Comparison between NMR mud logging result and well test result.

\begin{tabular}{|c|c|c|c|c|c|c|c|}
\hline \multirow{2}{*}{$\begin{array}{l}\text { Depth } \\
\text { (m) }\end{array}$} & \multirow{2}{*}{$\begin{array}{l}\text { Sample } \\
\text { No. }\end{array}$} & \multicolumn{4}{|c|}{$\begin{array}{l}\text { NMR mud logging result } \\
\text { (average value) }\end{array}$} & \multicolumn{2}{|c|}{ Test result $\left(\mathrm{m}^{3} / \mathrm{d}\right)$} \\
\hline & & $\begin{array}{c}\Phi \\
(\%)\end{array}$ & $\begin{array}{c}\mathrm{K} \\
(\mathrm{mD})\end{array}$ & $\begin{array}{l}\mathrm{Sm} \\
(\%)\end{array}$ & $\begin{array}{l}\text { So } \\
(\%)\end{array}$ & Oil & Water \\
\hline $\begin{array}{r}1970 \\
-1973\end{array}$ & 3 & 10.74 & 1.99 & 43.2 & 35.2 & 6.4 & 5.7 \\
\hline $\begin{array}{c}2379 \\
-2383\end{array}$ & 3 & 7.78 & 0.29 & 35.2 & 5.01 & 0.004 & 11.4 \\
\hline
\end{tabular}

porosity, the permeability, movable fluid saturation and oil saturation and so on.

At the interval 1970-1973m, the porosity, permeability, movable fluid saturation and oil saturation obtained by NMR logging are all higher, but the movable fluid saturation is $8 \%$ higher than the oil saturation (shown in Table 1). It is estimated that there were certain amount of movable water in the formation. Therefore, NMR logging interpreted this interval as oil with water. NMR mud logging interpretation coincides with test result.

At the interval $2379-2383 \mathrm{~m}$, the movable fluid saturation obtained by NMR logging is higher, but the oil saturation is very low, thus it can be estimated that the oil content in the formation is very small, the movable water is higher, so this layer is interpreted as water zone. The cuttings NMR logging explanatory result also coincides well with oil test result.

\section{Conclusions and Suggestion}

A. NMR mud logging can provide porosity, permeability, oil saturation, movable fluid saturation, irreducible water saturation, movable water saturation with high precision from rock sample. It can provide reliable data for the well completion discussion and drilling completion test, so it plays great role in the oil and gas exploration and production.
B. NMR mud logging can analyze the rock sample (core, cuttings, sidewall coring) on the well site.

C. In addition to evaluating petrophysical parameters, NMR mud logging can be used to classify and evaluate the pay zone.

D. NMR mud logging tests have been carried out in various oil fields in China, Sudan and Kazakhstan; The results show that NMR mud logging technology can provide reliable petrophysical parameters timely and accurately.

\section{REFERENCES}

[1] M. Appel, "Nuclear Magnetic Resonance and Formation Porosity: Petrophysics," Vol. 45, 2004, pp. 296-307.

[2] B. Blümich, J. Mauler, A. Haber, et al., "Mobile NMR for Geophysical Analysis and Materials Testing," Petroleum Science, Vol. 6, 2009, pp. 1-7

[3] G. R. Coates, L. Z. Xiao and M. G. Prammer, "NMR Logging Principles and Applications," Halliburton Energy Services, Houston: Gulf Publishing Company, 1999.

[4] R. Freedman and N. Heaton, "Fluid Characterization using Nuclear Magnetic Resonance Logging: Petrophysics," Vol. 45, 2004, pp. 241-250.

[5] Y. D He, Z. Q. Mao, L. Z. Xiao and X. J. Ren, "An Improved Method of using T2 Distribution to Evaluate Pore," Size Distribution: Chinese Journal of Geophysics, Vol. 2, pp. 373-378.

[6] K. Mirotchnik, S. Kryuchkov and K. Strack, "A Novel Method to Determine NMR Petrophysical Parameters," from Cuttings: SPWLA 45th Annual Logging Symposium.

[7] Y. T. Wang, M. H. Song and X. R. Cao, "Application of the NMR Mud Logging in Oilfield Exploration: China Petroleum Exploration," Vol. 4, pp. 66-70.

[8] Z. Z. Wang, M. Y. Deng, S. D. Zhai, et al., "Factors Affecting NMR T2 Spectra of oil Well Samples and Methods for Determining the T2 Cutoff Value," Chinese Journal of Magnetic Resonance, Vol. 1, pp. 143-151. 